\title{
Quality of life with second or third line nab-paclitaxel-based regimens in advanced non-small-cell lung cancer
}

\author{
Santiago Ponce Aix ${ }^{*} 1$, Denis Talbot ${ }^{2}$, Ramaswamy Govindan ${ }^{3}$, Manuel Cobo Dols ${ }^{4}$, Pieter E \\ Postmus ${ }^{5}$, Conrad Lewanski ${ }^{6}$, Jaafar Bennouna ${ }^{7}$, Juergen R Fischer ${ }^{8}$, Oscar Juan-Vidal ${ }^{9}$, \\ David J Stewart ${ }^{10}$, Andrea Ardizzoni ${ }^{11}$, Rafia Bhore ${ }^{12}$, Marianne Wolfsteiner ${ }^{13}$, Martin \\ Reck $^{14}$, Teng Jin Ong ${ }^{12}$ \& Daniel Morgensztern ${ }^{3}$ \\ ${ }^{1}$ Department of Medical Oncology, Hospital Universitario 12 de Octubre, Madrid, Spain \\ ${ }^{2}$ Department of Medical Oncology, Oxford University Hospitals NHS Foundation Trust, Oxfordshire, UK \\ ${ }^{3}$ Department of Medicine, Washington University School of Medicine, St Louis, MO 63110, USA \\ ${ }^{4}$ Department of Medical Oncology, Hospital Regional Universitario de Málaga, IBIMA, Málaga, Spain \\ ${ }^{5}$ Department of Medical Oncology, The Clatterbridge Cancer Centre, Liverpool, UK \\ ${ }^{6}$ Department of Clinical Oncology, Imperial College Healthcare NHS Trust, London, UK \\ ${ }^{7}$ Department of Medical Oncology, Centre René Gauducheau Centre de Lutte Contre Le Cancer Nantes Atlantique, Nantes, \\ Loire-Atlantique, France \\ ${ }^{8}$ Department of Medical Oncology, Lungenklinik Löwenstein gGmbH, Löwenstein, Baden-Württemberg, Germany \\ ${ }^{9}$ Department of Medical Oncology, Hospital Universitari i Politècnic La Fe, Valencia, Spain \\ ${ }^{10}$ Department of Medical Oncology, Ottawa Hospital, Ottawa, ON, Canada \\ ${ }^{11}$ Department of Medical Oncology, Azienda Ospedaliero Universitaria Di Bologna, Policlinico S Orsola-Malpighi, Bologna, Italy \\ ${ }^{12}$ Bristol-Myers Squibb, Summit, NJ 07901, USA \\ ${ }^{13}$ PRA Health Sciences, Lenexa, KS 66219, USA \\ ${ }^{14}$ LungenClinic, Airway Research Center North, German Center for Lung Research, Grosshansdorf, Germany \\ *Author for correspondence: Tel.: +34 914692 313; Fax: +34 914603 310; sponceaix@gmail.com
}

\begin{abstract}
Aim: Evaluate quality of life (QoL) in patients with advanced non-small cell lung cancer treated with second or third line nab-paclitaxel \pm durvalumab. Patients \& methods: Longitudinal QoL was assessed using Lung Cancer Symptom Scale, EuroQoL Five-Dimensions Five-Levels and European Organisation for Research and Treatment of Cancer Quality of Life Questionnaire 30-item core. Results: QoL was generally stable through eight treatment cycles (both arms). Clinically meaningful improvement from baseline was noted in Lung Cancer Symptom Scale (overall constitutional score and three-item index [nab-paclitaxel + durvalumab]) and European Organisation for Research and Treatment of Cancer Quality of Life Questionnaire 30-item core (global health status/QoL and emotional functioning [both arms] and pain [nab-paclitaxel + durvalumab]) analyses. EuroQoL Five-Dimensions Five-Levels domains were stable/improved or completely resolved at least once in $19-56 \%$ and $9-51 \%$ of patients, respectively. Conclusion: While QoL trends were promising, additional data are required to support these regimens in this setting.
\end{abstract}

First draft submitted: 3 December 2019; Accepted for publication: 6 March 2020; Published online: 31 March 2020

Keywords: durvalumab • EORTC QLQ-C30 • EQ-5D-5L • lung cancer symptom scale • nab-paclitaxel • non-small cell lung cancer $\bullet$ quality of life

Patients with advanced non-small cell lung cancer (NSCLC) have a high symptom burden that adversely affects their quality of life (QoL), which predictably worsens with the greater symptom burden associated with advanced disease $[1,2]$. In addition, QoL has been shown to significantly worsen with disease progression in patients with advanced NSCLC [3,4]. Therefore, QoL maintenance during second-line therapy for NSCLC is a valuable consideration for treatment decisions.

nab-Paclitaxel is currently approved in combination with carboplatin for frontline treatment of advanced NSCLC [5]. In the first-line setting, nab-paclitaxel + carboplatin has demonstrated clinically meaningful improvement in QoL from baseline [6]. Immune checkpoint inhibitors (ICIs) can improve outcomes with chemother-

Future Medicine 
apy [7-12], including nab-paclitaxel [10-12], in first-line treatment of advanced NSCLC. Nivolumab, pembrolizumab and atezolizumab are currently approved for second-line treatment of NSCLC [13]. Additionally, durvalumab as monotherapy has been shown to improve patient outcomes [14] and according to National Comprehensive Cancer Network guidelines, is a category 1 recommendation as consolidation treatment for patients with unresectable stage III disease that responded to platinum-based chemoradiation [15]. Additionally, targeted therapies have shown favorable QoL profiles [16-18]. However, to our knowledge, few studies have evaluated QoL, especially longitudinal QoL, in patients receiving second or third-line treatment.

The Phase II ABOUND.2L+ trial (NCT02250326) investigated treatment with second or third line nabpaclitaxel either alone or in combination with CC-486 (oral azacitidine) or durvalumab in patients with advanced NSCLC. The primary outcome of ABOUND.2L+ demonstrated no benefit of combining CC-486 with nabpaclitaxel; the median progression-free survival (PFS) was 3.2 months with nab-paclitaxel + CC-486 versus 4.2 months with nab-paclitaxel alone (hazard ratio: 1.3; 95\% CI: 0.9-1.9) [19]. In the third arm of the study, the combination of nab-paclitaxel with durvalumab demonstrated a median PFS of 4.5 months [20]. The objective of this analysis was to evaluate QoL data collected from the nab-paclitaxel alone and nab-paclitaxel + durvalumab arms, which demonstrated promising efficacy outcomes $[19,20]$. QoL outcomes with nab-paclitaxel + CC-486 were not reported because the regimen was found to be ineffective in this setting.

\section{Patients \& methods}

\section{Study design}

The study was approved by the institutional review board or independent ethics committee at participating sites and conducted in accordance with the principles of Good Clinical Practice and the Declaration of Helsinki. All patients provided written informed consent prior to treatment initiation.

Inclusion and exclusion criteria as well as sample size determination for the randomized part of this open-label study were described previously [19]. Briefly, patients with advanced nonsquamous NSCLC, one prior platinumbased chemotherapy and no activating EGFR mutations or $A L K$ translocations were randomized 1:1 to receive nab-paclitaxel $100 \mathrm{mg} / \mathrm{m}^{2}$ on days 8 and $15+$ CC- $486200 \mathrm{mg}$ on days $1-14$ or $n a b$-paclitaxel $100 \mathrm{mg} / \mathrm{m}^{2}$ on days 1 and 8 of each 21-day cycle. Randomization, when conducted, was performed centrally using a permuted-block randomization method. After enrollment for the nab-paclitaxel alone and nab-paclitaxel + CC-486 arms was completed, the ABOUND.2L+ protocol was amended to include a third arm, in which patients with advanced nonsquamous or squamous NSCLC and one prior platinum-based chemotherapy were enrolled. Patients were assigned to this third arm and received nab-paclitaxel $100 \mathrm{mg} / \mathrm{m}^{2}$ on days 1 and $8+$ durvalumab $1125 \mathrm{mg}$ on day 15 of a 21-day cycle. Hence, randomization did not occur between the nab-paclitaxel + durvalumab and nab-paclitaxel alone arms. The primary end point was PFS. Key secondary end points were overall survival, response rates and safety. QoL was an exploratory end point.

\section{QoL assessments}

QoL questionnaires were completed by patients on day 1 of each cycle. Assessments were conducted using the Lung Cancer Symptom Scale (LCSS), EuroQoL Five-Dimensions Five-Levels (EQ-5D-5L) and European Organisation for Research and Treatment of Cancer Quality of Life Questionnaire 30-item core (EORTC QLQ-C30), which have been previously validated [21-23].

The LCSS has nine components: appetite, fatigue, cough, dyspnea, hemoptysis, pain, lung cancer symptoms, normal activity and global QoL. Each item is measured on a $100-\mathrm{mm}$ visual analog scale (VAS). The LCSS composite scores include total score (average of all component scores), symptom burden index (average of appetite, fatigue, cough, dyspnea, hemoptysis and pain scores), pulmonary symptom scale (average of cough, dyspnea and hemoptysis scores), overall constitutional score (average of appetite and fatigue scores) and three-item index (sum of lung cancer symptoms, normal activity and global QoL scores; this index is measured on a 300-mm VAS). The LCSS total scores (0-100) were transformed by subtracting from 100 such that in the final outcome, a higher LCSS total score represents better health status and QoL.

The EQ-5D-5L measures five dimensions: mobility, self-care, usual activities, pain/discomfort and anxiety/depression. Readouts include utility and VAS scores (a higher score represents better health status).

The EORTC QLQ-C30 is a 30-item assessment with readouts including global health status/QoL, physical functioning, role functioning, emotional functioning, cognitive functioning, social functioning, fatigue, nausea 


\begin{tabular}{|c|c|}
\hline Characteristic & $n a b-P(n=80)$ \\
\hline \multicolumn{2}{|l|}{ Age (years) } \\
\hline$\geq 65$ years & $39(48.8)$ \\
\hline$\geq 70$ years & $19(23.8)$ \\
\hline$\geq 75$ years & $10(12.5)$ \\
\hline Male & $50(62.5)$ \\
\hline Female & $30(37.5)$ \\
\hline \multicolumn{2}{|l|}{ Race } \\
\hline White & $63(78.8)$ \\
\hline Black or African American & $2(2.5)$ \\
\hline \multicolumn{2}{|l|}{ ECOG PS } \\
\hline 0 & $26(32.5)$ \\
\hline 1 & $54(67.5)$ \\
\hline \multicolumn{2}{|l|}{ Histology } \\
\hline Squamous & 0 \\
\hline Nonsquamous & $80(100)$ \\
\hline Not specified & 0 \\
\hline
\end{tabular}

and vomiting and pain. For global health status/QoL and functioning scales, a higher score represents better health status. For symptom scales, a lower score represents better health status.

For items measured on a VAS, improvement $\geq 10 \mathrm{~mm}$ was considered clinically meaningful [24]. For the LCSS three-item index, improvement $\geq 30 \mathrm{~mm}$ was considered clinically meaningful [25].

Patients with QoL data at baseline and $\geq 1$ postbaseline assessment were included. Data cutoffs were 30 August 2017 for the nab-paclitaxel alone arm and 23 December 2017 for the nab-paclitaxel + durvalumab arm.

\section{Results}

\section{Baseline patient characteristics}

The ABOUND.2L + trial included 80 patients randomized to the $n a b$-paclitaxel alone arm and 79 patients assigned to the nab-paclitaxel + durvalumab arm. The baseline demographics by treatment arm are reported in Table 1 and 2. Notable differences were that the nab-paclitaxel + durvalumab arm included patients with both squamous and nonsquamous histology (the nab-paclitaxel alone arm included only patients with nonsquamous NSCLC) and had a higher frequency of patients with an Eastern Cooperative Oncology Group performance status (ECOG PS) of 1 (77.2 vs $67.5 \%$ for nab-paclitaxel + durvalumab vs nab-paclitaxel alone).

\section{QoL assessment completion}

A total of $55(68.8 \%)$ and $69(87.3 \%)$ patients completed a baseline QoL assessment in the nab-paclitaxel alone and nab-paclitaxel + durvalumab arms, respectively. However, a total of $50(62.5 \%)$ and $58(73.4 \%)$ patients, respectively, completed a baseline and $\geq 1$ postbaseline QoL assessment.

\section{LCSS results}

The LCSS total score (represented as mean change from baseline) was stable through eight cycles of treatment in both arms, with no clinically meaningful changes during the first eight cycles (Figure 1A \& B). The mean maximum change from baseline showed stable QoL in all five LCSS composite scores in the nab-paclitaxel alone arm (Figure 2A), and in three of the five composite scores (total score, symptom burden index and pulmonary 
Table 2. Baseline patient characteristics for patients who received nab-paclitaxel + durvalumab.

\begin{tabular}{|c|c|}
\hline Characteristic & $n a b-P+D(n=79)$ \\
\hline \multicolumn{2}{|l|}{ Age (years) } \\
\hline Median (range) & $63.0(29-84)$ \\
\hline$\geq 65$ years & $36(45.6)$ \\
\hline$\geq 70$ years & $25(31.6)$ \\
\hline$\geq 75$ years & $9(11.4)$ \\
\hline \multicolumn{2}{|l|}{ Sex } \\
\hline Female & $25(31.6)$ \\
\hline \multicolumn{2}{|l|}{ Race } \\
\hline White & $77(97.5)$ \\
\hline Black or African American & $1(1.3)$ \\
\hline Asian & 0 \\
\hline 1 & $61(77.2)$ \\
\hline \multicolumn{2}{|l|}{ Histology } \\
\hline Squamous & $23(29.1)$ \\
\hline Nonsquamous & 55 (69.6) \\
\hline Not specified & $1(1.3)$ \\
\hline
\end{tabular}

symptoms) in the nab-paclitaxel + durvalumab arm (Figure 2B). In addition, clinically meaningful improvement was noted in the three-item index and overall constitutional score in the nab-paclitaxel + durvalumab arm.

LCSS component and composite scores were stable through the first eight cycles of treatment in both arms (Table $3 \& 4$ ), with a few exceptions observed primarily in the nab-paclitaxel alone arm. Among patients who received nab-paclitaxel alone, clinically meaningful improvement occurred in the cough item in several cycles, and in dyspnea, global QoL and pulmonary symptoms at cycle 7; clinically meaningful deterioration occurred in fatigue, hemoptysis, lung cancer symptoms, normal activity and overall constitutional score at cycle 8 (Table 3). Interestingly, the mean maximum scores showed clinically meaningful improvement in seven of the nine items in the nab-paclitaxel alone arm, and in eight of the nine items in the nab-paclitaxel + durvalumab arm; the scores for hemoptysis in both arms and lung cancer symptoms in the nab-paclitaxel alone arm were stable.

\section{EQ-5D-5L results}

EQ-5D-5L VAS score (represented as mean change from baseline) was stable through eight cycles of treatment in both arms (Figure 3A \& B), with the exception of a clinically meaningful deterioration noted in the nab-paclitaxel alone arm at cycle 8 . The domains of mobility, self-care, usual activities, pain/discomfort and anxiety/depression were stable or improved from baseline in $29-56 \%$ (Figure $4 \mathrm{~A}$ ) and $19-48 \%$ (Figure 4B) of patients in the nabpaclitaxel alone and nab-paclitaxel + durvalumab arms, respectively. The domains were completely resolved at least once during treatment in 9-39\% (Figure 4C) and 31-51\% (Figure 4D) of patients in the nab-paclitaxel alone and nab-paclitaxel + durvalumab arms, respectively.

\section{EORTC QLQ-C30 results}

EORTC QLQ-C30 scores were generally stable through the first eight cycles of treatment in both arms, with the exception of clinically meaningful deterioration in role functioning at cycles 6 and 8 with nab-paclitaxel alone (Table 5) and at cycle 8 with nab-paclitaxel + durvalumab (Table 6), and improvement in emotional and cognitive functioning beginning with cycles 4 and 5, respectively, as well as social functioning at cycle 7 in the nab-paclitaxel alone arm. Mean maximum EORTC QLQ-C30 scores showed clinically meaningful improvement in emotional 
Table 3. Mean change from baseline over time and mean maximum improvement from baseline in patients treated with nab-paclitaxel alone (Lung Cancer Symptom Scale component and composite).

\begin{tabular}{|c|c|c|c|c|c|c|c|c|c|}
\hline Assessment & $\begin{array}{l}\text { C2,D1 } \\
(n=42)\end{array}$ & $\begin{array}{l}\text { C3,D1 } \\
(n=33)\end{array}$ & $\begin{array}{l}\text { C4,D1 } \\
(n=29)\end{array}$ & $\begin{array}{l}\text { C5,D1 } \\
(n=23)\end{array}$ & $\begin{array}{l}\text { C6,D1 } \\
(n=19)\end{array}$ & $\begin{array}{l}C 7, D 1 \\
(n=12)\end{array}$ & $\begin{array}{l}\text { C8,D1 } \\
(n=12)\end{array}$ & $\begin{array}{l}\text { C9,D1 } \\
(n=10)\end{array}$ & $\begin{array}{l}\text { Max } \\
(n=50)\end{array}$ \\
\hline \multicolumn{10}{|c|}{ LCSS component } \\
\hline Appetite & -2.3 & 2.0 & 6.9 & 2.5 & 0.7 & 8.8 & -9.8 & -1.7 & 11.5 \\
\hline Fatigue & -3.7 & -1.8 & 3.2 & 1.1 & -8.6 & 7.8 & -13.2 & -13.2 & 10.9 \\
\hline Cough & 1.9 & 8.1 & 15.0 & 11.0 & 6.2 & 20.8 & 5.1 & 10.2 & 16.8 \\
\hline Dyspnea & -1.6 & 2.6 & 7.9 & 6.8 & 3.6 & 13.2 & -7.0 & 6.9 & 11.8 \\
\hline Hemoptysis & 2.6 & 1.9 & 0.4 & -3.8 & -6.1 & -1.8 & -15.4 & -7.4 & 5.5 \\
\hline Pain & -1.3 & -4.5 & 4.9 & 5.2 & -1.7 & 8.4 & -6.9 & -6.0 & 11.5 \\
\hline $\begin{array}{l}\text { Lung cancer } \\
\text { symptoms }\end{array}$ & -0.1 & 3.5 & -0.3 & 3.3 & -7.4 & 5.5 & -14.3 & -3.7 & 9.7 \\
\hline $\begin{array}{l}\text { Normal } \\
\text { activity }\end{array}$ & 1.0 & -5.2 & 1.1 & -4.7 & -7.5 & 4.5 & -12.2 & -17.0 & 12.1 \\
\hline Global QoL & -0.3 & 6.1 & 5.6 & 7.4 & 5.2 & 14.8 & 5.4 & -3.7 & 12.2 \\
\hline \multicolumn{10}{|l|}{ LCSS composite } \\
\hline $\begin{array}{l}\text { Pulmonary } \\
\text { symptoms }\end{array}$ & 1.0 & 4.2 & 7.8 & 4.7 & 1.2 & 10.7 & -5.8 & 3.2 & 9.6 \\
\hline $\begin{array}{l}\text { Three-item } \\
\text { index } \ddagger\end{array}$ & 0.6 & 4.5 & 6.4 & 6.0 & -9.7 & 24.8 & -21.1 & -24.4 & 25.0 \\
\hline $\begin{array}{l}\text { Symptom } \\
\text { burden index }\end{array}$ & -0.7 & 1.4 & 6.4 & 3.8 & -1.0 & 9.5 & -7.9 & -1.9 & 7.9 \\
\hline $\begin{array}{l}\text { Overall } \\
\text { constitutional } \\
\text { score } \mathbb{I}\end{array}$ & -3.0 & 0.1 & 5.1 & 1.8 & -3.9 & 8.3 & -11.5 & -7.5 & 9.5 \\
\hline \multicolumn{10}{|c|}{ 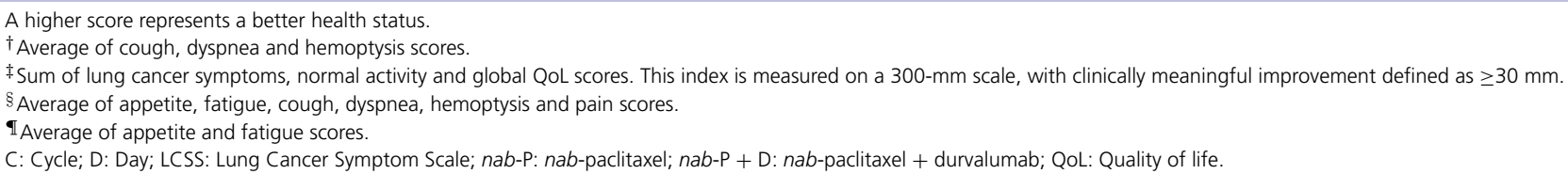 } \\
\hline
\end{tabular}

Table 4. Mean change from baseline over time and mean maximum improvement from baseline in patients treated with nab-paclitaxel + durvalumab (Lung Cancer Symptom Scale component and composite).

\begin{tabular}{|c|c|c|c|c|c|c|c|c|c|}
\hline Assessment & $\begin{array}{l}\text { C2,D1 } \\
(n=52)\end{array}$ & $\begin{array}{l}\text { C3,D1 } \\
(n=49)\end{array}$ & $\begin{array}{l}\text { C4,D1 } \\
(n=43)\end{array}$ & $\begin{array}{l}\text { C5,D1 } \\
(n=43)\end{array}$ & $\begin{array}{l}\text { C6,D1 } \\
(n=37)\end{array}$ & $\begin{array}{l}\text { C7,D1 } \\
(n=35)\end{array}$ & $\begin{array}{l}\text { C8,D1 } \\
(n=31)\end{array}$ & $\begin{array}{l}\text { C9,D1 } \\
(n=23)\end{array}$ & $\begin{array}{l}\text { Max } \\
(n=58)\end{array}$ \\
\hline \multicolumn{10}{|l|}{ LCSS component } \\
\hline Appetite & 1.4 & -1.0 & 2.9 & 6.3 & 5.2 & -3.5 & 2.8 & 4.7 & 16.0 \\
\hline Fatigue & -3.0 & -2.0 & 1.8 & 7.6 & -1.8 & 0.1 & 0.1 & -4.2 & 15.2 \\
\hline Cough & -5.1 & -3.1 & 4.2 & 5.0 & 6.2 & 2.9 & 3.0 & 6.7 & 14.7 \\
\hline Dyspnea & -4.1 & -1.3 & -1.3 & 2.8 & 0.7 & -4.3 & 2.0 & -2.2 & 11.5 \\
\hline Hemoptysis & 2.2 & 0.6 & 2.8 & 4.0 & 1.5 & 0.8 & 4.7 & -0.3 & 5.5 \\
\hline Pain & 5.3 & 2.4 & -1.6 & 5.6 & 8.0 & 7.3 & 2.4 & 9.0 & 15.1 \\
\hline Lung cancer symptoms & 2.9 & 2.0 & 0.1 & 5.3 & 12.5 & 7.6 & 6.1 & 5.9 & 16.4 \\
\hline Normal activity & -4.5 & -1.7 & 1.3 & 2.7 & 0.8 & -4.1 & -4.8 & -2.2 & 11.3 \\
\hline Global QoL & -1.5 & -4.7 & -1.3 & 0.4 & -3.5 & -6.1 & -9.2 & -5.2 & 10.9 \\
\hline \multicolumn{10}{|l|}{ LCSS composite } \\
\hline Pulmonary symptoms ${ }^{\dagger}$ & -2.3 & -1.3 & 1.9 & 3.9 & 2.8 & -0.2 & 3.2 & 1.4 & 9.2 \\
\hline Three-item index $\ddagger$ & -3.1 & -4.4 & 0.1 & 8.5 & 9.7 & -2.7 & -7.9 & -1.6 & 30.4 \\
\hline Symptom burden index $\S$ & -0.5 & -0.7 & 1.5 & 5.2 & 3.3 & 0.5 & 2.5 & 2.3 & 9.3 \\
\hline Overall constitutional score $\mathbb{I}$ & -0.8 & -1.5 & 2.3 & 7.0 & 1.7 & -1.7 & 1.5 & 0.3 & 13.0 \\
\hline \multicolumn{10}{|c|}{$\begin{array}{l}\text { A higher score represents a better health status. } \\
\text { †Average of cough, dyspnea and hemoptysis scores. } \\
\text { ¥Sum of lung cancer symptoms, normal activity and global QoL scores. This index is measured on a 300-mm scale, with clinically meaningful improvement defined as } \geq 30 \text { mm. } \\
\text { §Average of appetite, fatigue, cough, dyspnea, hemoptysis and pain scores. } \\
\mathbb{I}_{\text {Average of appetite and fatigue scores. }} \\
\text { C: Cycle; D: Day; LCSS: Lung Cancer Symptom Scale; nab-P: nab-paclitaxel; nab-P + D: nab-paclitaxel + durvalumab; QoL: Quality of life. }\end{array}$} \\
\hline
\end{tabular}


(A)

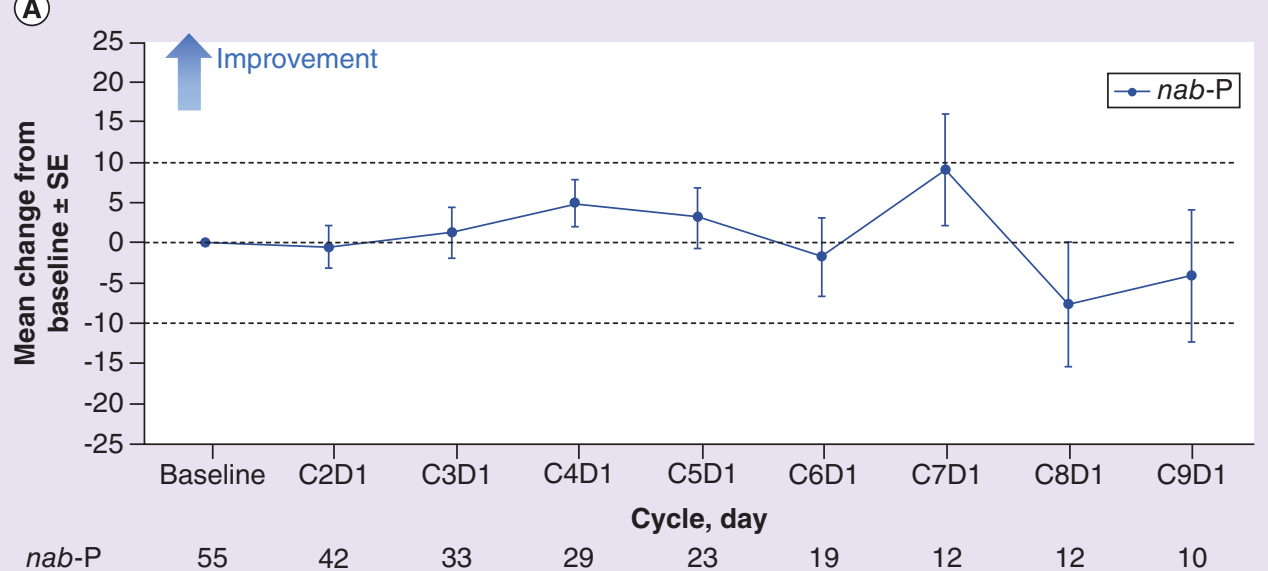

(B)

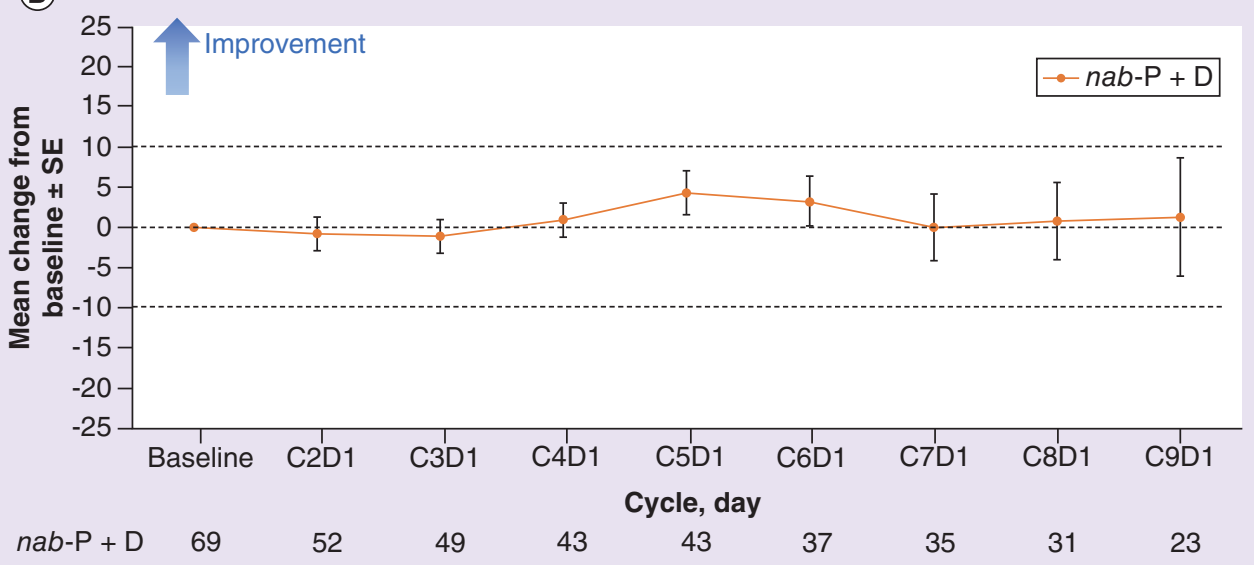

Figure 1. Change from baseline in quality of life (Lung Cancer Symptom Scale total score ${ }^{\dagger}$ ). (A) Patients who received nab-P alone and (B) Patients who received nab-P $+\mathrm{D}$. A higher score represents a better health status.

${ }^{\dagger}$ Average of appetite, fatigue, cough, dyspnea, hemoptysis, pain, lung cancer symptoms, normal activity and global QoL scores.

C: Cycle; D: Day; nab-P: nab-paclitaxel; nab-P + D: nab-paclitaxel + durvalumab; QoL: Quality of life.

functioning and global health status/QoL in both arms (Figure 5A \& B), and in pain (decreased score denotes improvement) in the nab-paclitaxel + durvalumab arm. Physical, role, cognitive and social functioning, as well as fatigue and nausea/vomiting were stable in both arms.

\section{Performance status deterioration}

Among those who received nab-paclitaxel alone and had a baseline ECOG PS 0, 18 (78.3\%) patients experienced a performance status deterioration of $\geq 1$; among those who had a baseline ECOG PS 1, 15 (26.3\%) patients experienced a performance status deterioration of $\geq 1$. Among those who received nab-paclitaxel + durvalumab and had a baseline ECOG PS 0, $15(83.3 \%)$ patients experienced a performance status deterioration of $\geq 1$; among those who had a baseline ECOG PS 1, $22(36.1 \%)$ patients experienced a performance status deterioration of $\geq 1$.

\section{Discussion}

The results of this analysis show that in general, QoL scores were stable or improved through the first eight cycles of treatment with two nab-paclitaxel-based regimens used in the ABOUND.2L+ study: nab-paclitaxel alone and nabpaclitaxel + durvalumab. Clinically meaningful improvement in mean maximum improvement occurred in both treatment arms in the LCSS components of fatigue, cough, dyspnea, appetite, pain, normal activity and global QoL. 


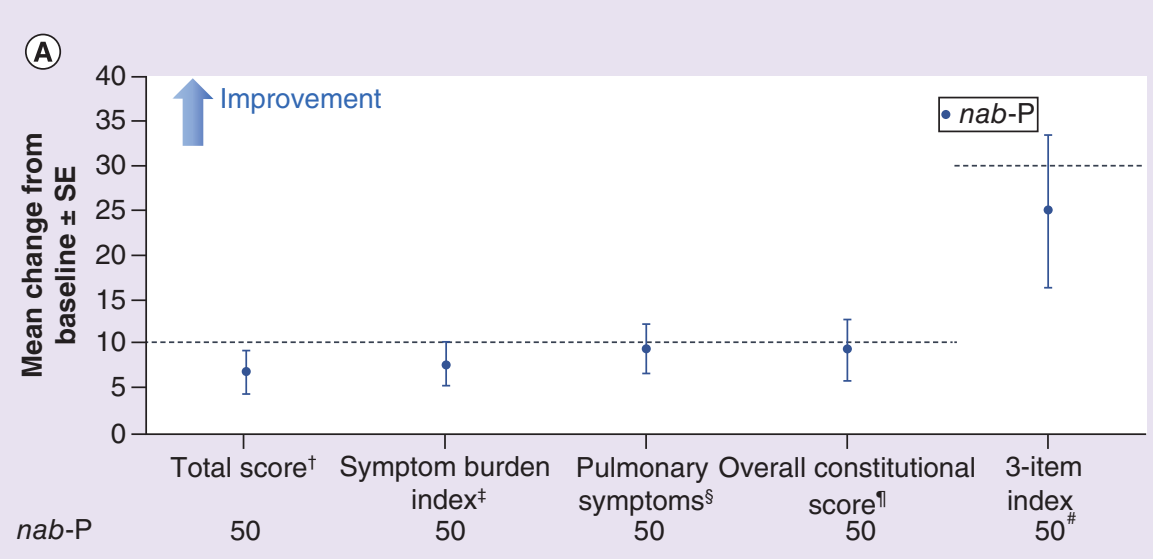

(B)

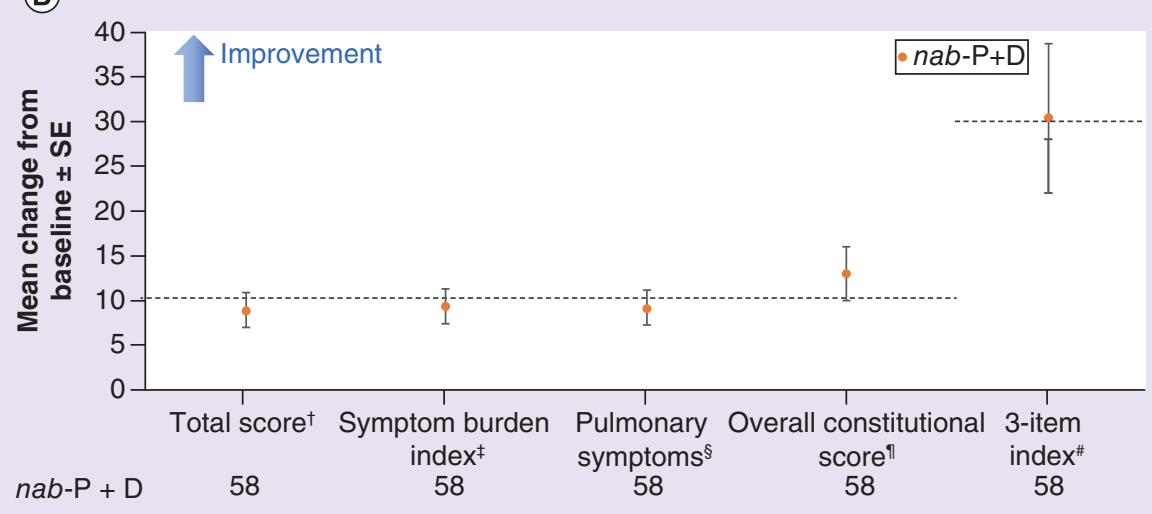

Figure 2. Improvement from baseline in Lung Cancer Symptom Scale composite scores (mean maximum). (A) Patients who received nab-P alone and (B) Patients who received nab-P $+\mathrm{D}$. A higher score represents a better health status.

${ }^{\dagger}$ Average of appetite, fatigue, cough, dyspnea, hemoptysis, pain, lung cancer symptoms, normal activity and global QoL scores.

${ }^{\ddagger}$ Average of appetite, fatigue, cough, dyspnea, hemoptysis and pain scores.

§average of cough, dyspnea and hemoptysis scores.

ISum of lung cancer symptoms, normal activity and global QoL scores. This item is measured on a 300-mm scale, with clinically meaningful improvement defined as $\geq 30 \mathrm{~mm}$.

\#Average of appetite and fatigue scores.

nab-P: nab-paclitaxel; nab-P + D: nab-paclitaxel + durvalumab; QoL: Quality of life.

Clinically meaningful improvement was also noted in lung cancer symptoms in the nab-paclitaxel + durvalumab arm. In the EQ-5D-5L domains, 19-56\% of patients reported stable or improved status, while $9-51 \%$ of patients reported complete resolution of a problem in a domain at least once. Clinically meaningful improvement in mean maximum improvement also occurred in EORTC QLQ-C30 emotional functioning and global health status/QoL (nab-paclitaxel alone and nab-paclitaxel + durvalumab) and pain (nab-paclitaxel + durvalumab) scores. The impact of nab-paclitaxel-based regimens on QoL observed in our study is supported by a previous report of nabpaclitaxel + carboplatin for frontline treatment of patients with squamous NSCLC, which reported stable QoL through four cycles of induction treatment based on LCSS scores, and stable or improved status in the EQ-5D-5L domains in $\geq 83 \%$ of patients [6].

Given the high symptom burden in patients with advanced NSCLC [2] as well as the significantly worse QoL associated with disease progression [3,4], it is important to consider the impact of treatment on specific symptoms as well as overall QoL and other clinical outcomes. The nab-paclitaxel-based regimens employed in this trial generally kept symptom severity, as assessed by LCSS component scores, stable throughout treatment, with little clinically meaningful deterioration. 

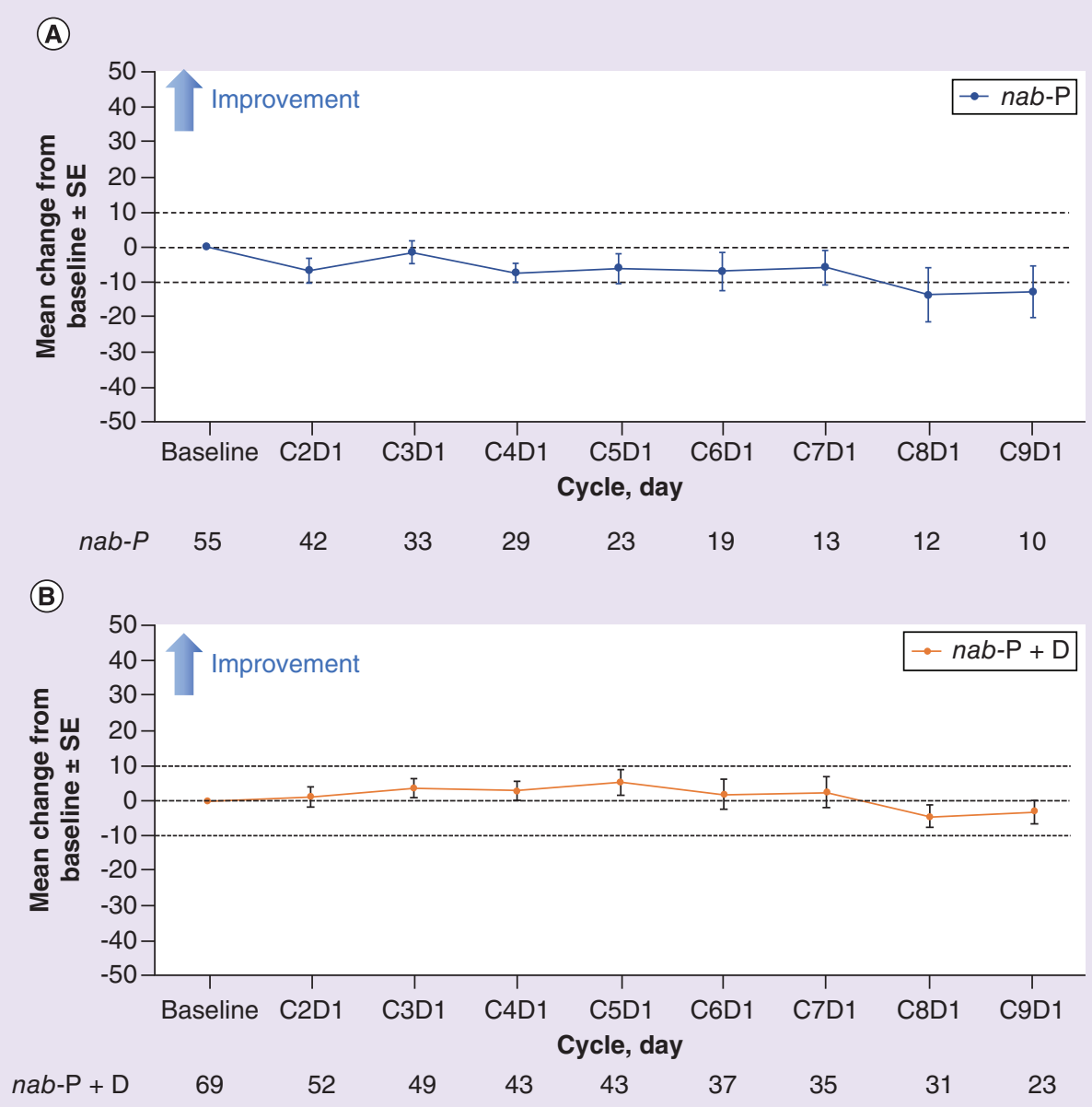

Figure 3. Change from baseline in quality of life (EuroQoL Five-Dimensions Five-Levels Visual Analog Scale score ${ }^{\dagger}$ ). (A) Patients who received nab-P alone and (B) Patients who received nab-P + D. A higher score represents a better health status.

†EQ-5D-5L VAS score records the patient's self-rated health on a VAS with end points 'The best health you can imagine' and 'The worst health you can imagine'. VAS score was calculated using the US Crosswalk Index Value set. C: Cycle; D: Day; EQ-5D-5L: EuroQoL Five-Dimensions Five-Levels; nab-P: nab-paclitaxel; nab-P + D: nab-paclitaxel + durvalumab; QoL: Quality of life; VAS: Visual analog scale.

Beyond the physical impact of NSCLC, the assessments reported here also examined the psychological and social impacts of the disease. Previous studies revealed the presence of psychological and social problems facing patients with NSCLC, including anxiety, lung cancer-related stigma and depression and have suggested an association between anxiety and depression with mortality in these patients [26-28]. In the current study, based on EORTC QLQ-C30, emotional functioning showed clinically meaningful improvement compared with baseline at several time points throughout the treatment in the $n a b$-paclitaxel alone arm and remained stable in the $n a b$ paclitaxel + durvalumab arm. Additionally, the anxiety/depression domain of the EQ-5D-5L was completely resolved at least once in 37 and $50 \%$ of patients in the nab-paclitaxel alone and nab-paclitaxel + durvalumab arms, respectively. Although our findings cannot be compared directly with those from previous studies, a possible explanation could be the promising activity with treatment, but such interpretations must be made cautiously due to potential survivor selection bias in longitudinal QoL analyses.

The current National Comprehensive Cancer Network guidelines for NSCLC include ICIs (nivolumab, pembrolizumab and atezolizumab) as category 1 , preferred subsequent therapy options for patients without prior ICI therapy [15]. In addition, docetaxel, pemetrexed (nonsquamous), gemcitabine, or ramucirumab plus docetaxel are category $2 \mathrm{~A}$ options. Given the major impact of ICIs plus chemotherapy in the first-line setting, there will con- 
(A)

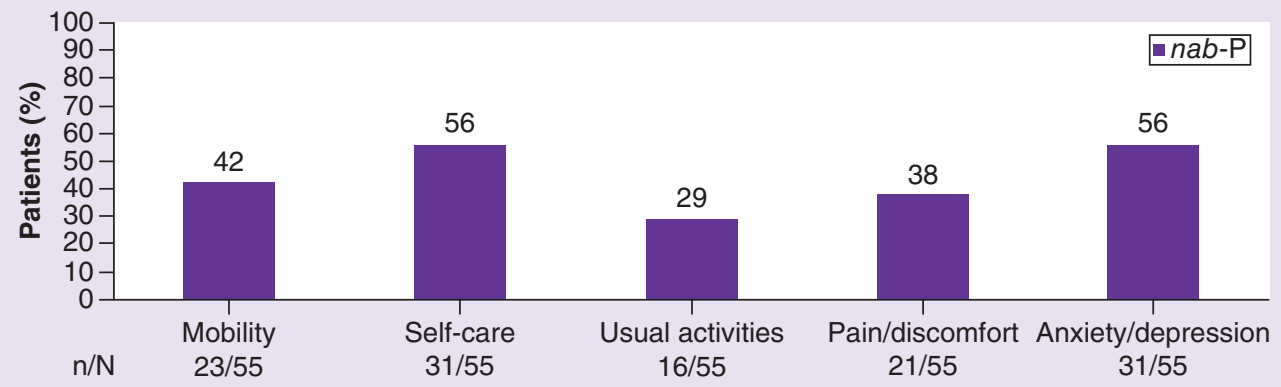

(B)

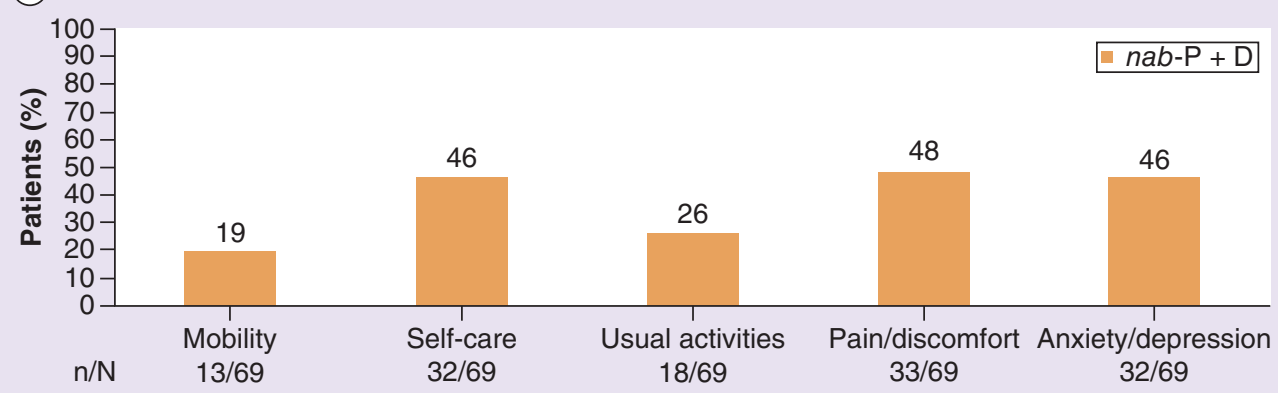

(C)

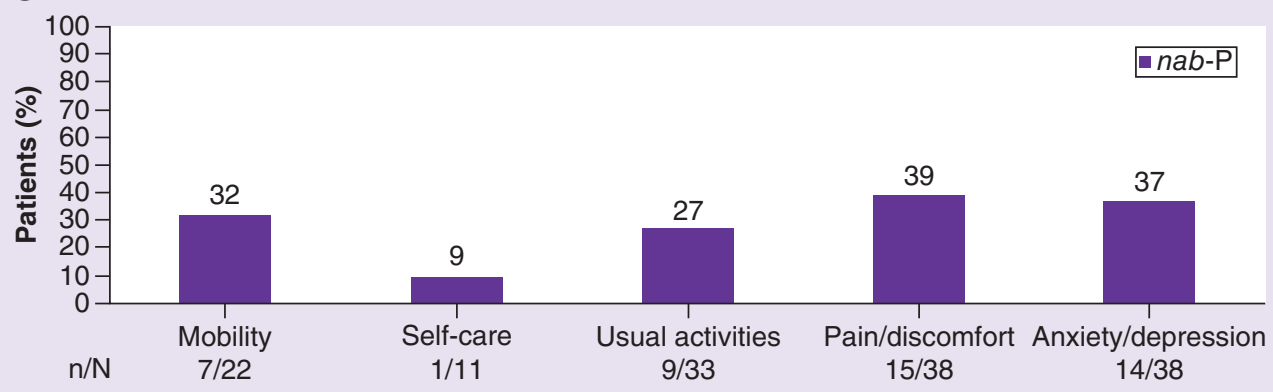

(D)

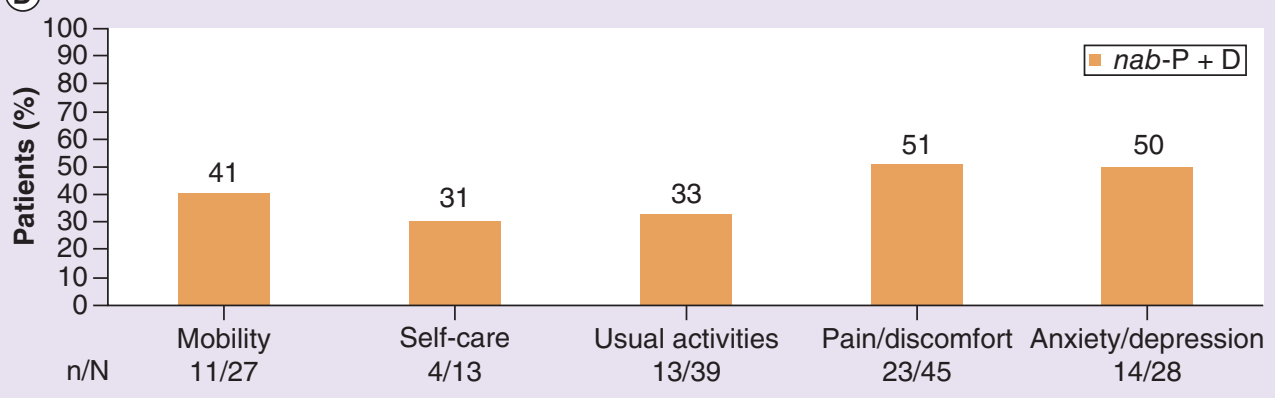

Figure 4. Resolution of problems ${ }^{\dagger}$ (EuroQoL Five-Dimensions Five-Levels). Stable or improved from baseline (nab-P alone $[\mathrm{A}]$ or nab-P $+\mathrm{D}[\mathrm{B}]$ ) or complete resolution of symptoms at least once (nab-P alone [C] or nab-P $+\mathrm{D}[\mathrm{D}]$ ). The percentage of patients with a stable or improved dimension is based on the population with a baseline assessment. The percentage of patients with a dimension completely resolved at least once is based on the population with a problem in the given dimension at baseline. (A) Stable or improved from baseline (nab-P alone). (B) Stable or improved from baseline (nab-P + D). (C) Complete resolution of symptoms at least once (nab-P alone). (D) Complete resolution of symptoms at least once (nab-P $+\mathrm{D})$.

$\dagger$ Patients rate their health state in each domain as 'no problems', 'slight problems', 'moderate problems', 'severe problem' and 'extreme problems'. A dimension is stable or improved if it stays the same or improves compared with baseline during the study. A dimension is completely resolved if the score becomes 'no problems'.

nab-P: nab-paclitaxel; nab-P + D: nab-paclitaxel + durvalumab. 


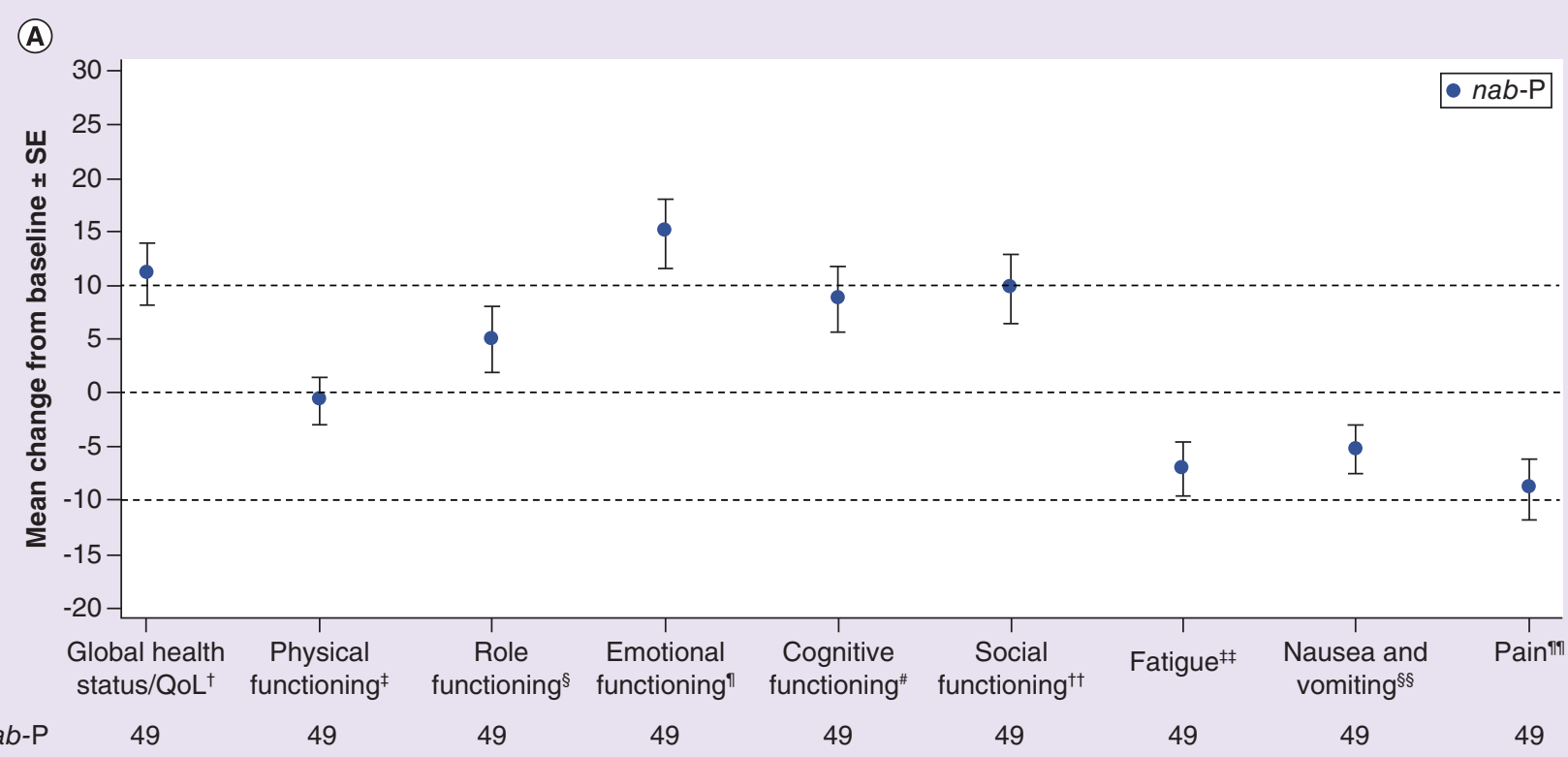

(B)

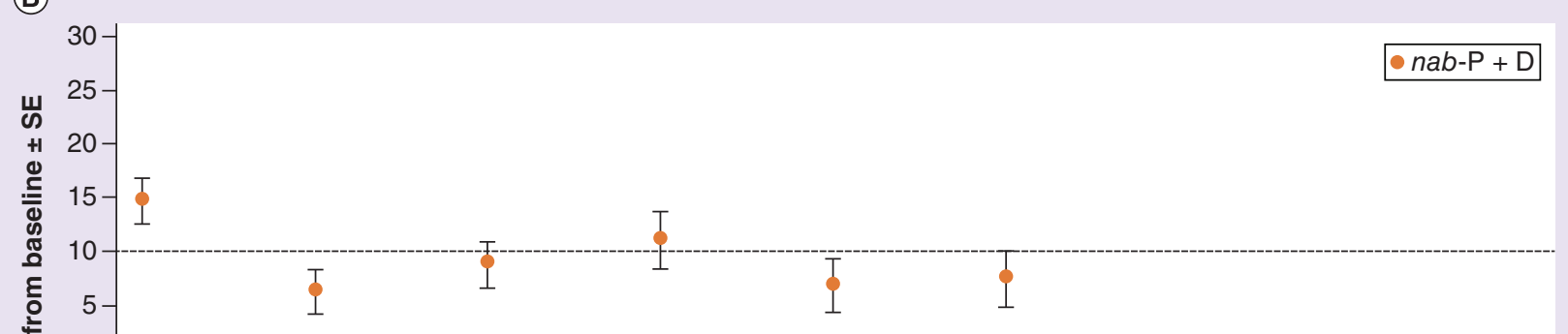

Figure 5. Improvement from baseline in European Organisation for Research and Treatment of Cancer Quality of Life Questionnaire 30-item core scores (mean maximum). (A) Patients who received nab-P alone and (B) Patients who received nab-P + D. For global health status/QoL and functioning scales, a higher score represents a better health status. For symptom scales, a lower score represents a better health status.

$\dagger$ Includes items 29 and 30 on the EORTC QLQ-C30. † § $\mathbb{I} \#$

$\ddagger$ Includes items $1-5$.

$\S$ Includes items 6 and 7.

Includes items 21-24.

\#Includes items 20 and 25.

$\dagger \dagger$ Includes items 26 and 27.

$\ddagger \ddagger$ Includes items 10, 12 and 18.

$\S \S$ Includes items 14 and 15 .

IIIncludes items 9 and 19.

EORTC QLQ-C30: European Organisation for Research and Treatment of Cancer Quality of Life Questionnaire 30-item core; nab-P: nab-paclitaxel; nab-P + D: nab-paclitaxel + durvalumab; QoL: Quality of life. 
Table 5. Mean change from baseline over time in patients treated with nab-paclitaxel alone (European Organisation for Research and Treatment of Cancer Quality of Life Questionnaire 30-item core).

\begin{tabular}{|c|c|c|c|c|c|c|c|c|}
\hline Assessment & $\begin{array}{l}\text { C2,D1 } \\
(n=42)\end{array}$ & $\begin{array}{l}\text { C3,D1 } \\
(n=33)\end{array}$ & $\begin{array}{l}\text { C4,D1 } \\
(n=29)\end{array}$ & $\begin{array}{l}\text { C5,D1 } \\
(n=23)\end{array}$ & $\begin{array}{l}\text { C6,D1 } \\
(n=19)\end{array}$ & $\begin{array}{l}\text { C7,D1 } \\
(n=12)\end{array}$ & $\begin{array}{l}\text { C8,D1 } \\
(n=12)\end{array}$ & $\begin{array}{l}\text { C9,D1 } \\
(n=10)\end{array}$ \\
\hline Global health status/QoL ${ }^{\dagger}$ & 0.60 & 7.07 & 1.72 & 1.81 & 0.00 & 0.00 & -1.39 & -6.67 \\
\hline Physical functioning $\ddagger$ & -4.44 & -3.03 & -5.98 & -3.48 & -6.32 & -0.56 & -8.89 & -16.67 \\
\hline Role functioning ${ }^{\S}$ & -2.38 & -7.58 & -7.47 & -5.80 & -10.53 & -4.17 & -13.89 & -21.67 \\
\hline Emotional functioning $\mathbb{I}$ & 5.56 & 7.32 & 10.34 & 14.86 & 18.42 & 17.36 & 10.42 & 6.67 \\
\hline Cognitive functioning ${ }^{\#}$ & 3.57 & 7.58 & 6.90 & 11.59 & 10.53 & 11.11 & 11.11 & 13.33 \\
\hline Social functioning ${ }^{\dagger \dagger}$ & 1.59 & 3.54 & -5.17 & 2.90 & -1.75 & 13.89 & 5.56 & 5.00 \\
\hline Fatigue & 3.44 & 5.05 & 6.13 & 1.45 & -1.17 & -3.70 & 2.78 & 5.56 \\
\hline Nausea and vomiting $\S \S$ & 0.79 & 1.01 & 2.87 & 0.72 & 3.51 & 1.39 & 6.94 & 8.33 \\
\hline Pain $\mathbb{I}$ & -0.40 & 0.00 & 2.30 & -7.97 & -0.88 & 2.78 & 9.72 & 15.00 \\
\hline
\end{tabular}

For global health status/QoL and functioning scales, a higher score represents a better health status. For symptom scales, a lower score represents a better health status.

$\dagger$ Includes items 29 and 30 on the EORTC QLQ-C30.

$¥$ Includes items $1-5$.

$\S$ Includes items 6 and 7

Includes items 21-24.

\#Includes items 20 and 25 .

$\dagger \dagger$ Includes items 26 and 27.

$\$$ \#ncludes items 10,12 and 18

$\S \S$ Includes items 14 and 15

$\mathbb{I}$ Includes items 9 and 19.

C: Cycle; D: Day; EORTC QLQ-C30, European Organisation for Research and Treatment of Cancer Quality of Life Questionnaire 30-item core; nab-P: nab-paclitaxel; nab-P + D: nabpaclitaxel + durvalumab; QoL: Quality of life.

Table 6. Mean change from baseline over time in patients treated with nab-paclitaxel + durvalumab (European Organisation for Research and Treatment of Cancer Quality of Life Questionnaire 30-item core).

\begin{tabular}{|c|c|c|c|c|c|c|c|c|}
\hline Assessment & $\begin{array}{l}\text { C2,D1 } \\
(n=52)\end{array}$ & $\begin{array}{l}\text { C3,D1 } \\
(n=49)\end{array}$ & $\begin{array}{l}\text { C4,D1 } \\
(n=43)\end{array}$ & $\begin{array}{l}\text { C5,D1 } \\
(n=43)\end{array}$ & $\begin{array}{l}\text { C6,D1 } \\
(n=37)\end{array}$ & $\begin{array}{l}\text { C7,D1 } \\
(n=35)\end{array}$ & $\begin{array}{l}\text { C8,D1 } \\
(n=31)\end{array}$ & $\begin{array}{l}\text { C9,D1 } \\
(n=23)\end{array}$ \\
\hline Global health status $/ \mathrm{QoL}^{\dagger}$ & 2.08 & 4.59 & 1.55 & 4.07 & 0.45 & -1.19 & -1.88 & 0.00 \\
\hline Physical functioning $\ddagger$ & -0.90 & -2.04 & 0.16 & -0.16 & -4.50 & -5.14 & -9.03 & -9.28 \\
\hline Role functioning ${ }^{\S}$ & -5.13 & -2.38 & -1.55 & -4.65 & -4.50 & -7.14 & -11.83 & -15.22 \\
\hline Emotional functioning $\mathbb{I}$ & 2.72 & 1.53 & 5.43 & 5.62 & 4.73 & 5.24 & 8.06 & 0.36 \\
\hline Cognitive functioning $\#$ & 1.60 & -0.34 & 0.78 & 2.71 & 0.00 & 3.81 & -0.54 & -2.17 \\
\hline Social functioning ${ }^{\dagger \dagger}$ & -4.49 & -5.78 & -1.55 & -2.71 & -1.80 & -4.76 & -5.38 & -2.17 \\
\hline Fatigue & 1.28 & 0.45 & 0.26 & -1.03 & 5.11 & 5.71 & 9.32 & 12.56 \\
\hline Nausea and vomiting ${ }^{\S \S}$ & -0.64 & 0.68 & -2.33 & -2.71 & -4.05 & -1.90 & 0.00 & -5.07 \\
\hline Pain $\mathbb{I}$ & -2.56 & -0.68 & 0.39 & -3.49 & -5.41 & -2.86 & 1.61 & 1.45 \\
\hline
\end{tabular}

For global health status/QoL and functioning scales, a higher score represents a better health status. For symptom scales, a lower score represents a better health status.

$\dagger$ Includes items 29 and 30 on the EORTC QLQ-C30.

¥Includes items $1-5$.

Includes items 6 and 7.

In Includes items 21-24.

\#Includes items 20 and 25.

$\dagger \dagger$ Includes items 26 and 27.

$\ddagger \ddagger$ Includes items 10,12 and 18 .

$\S \S$ Includes items 14 and 15 .

$\mathbb{I}$ Includes items 9 and 19.

C: Cycle; D: Day; EORTC QLQ-C30, European Organisation for Research and Treatment of Cancer Quality of Life Questionnaire 30-item core; nab-P: nab-paclitaxel; nab-P + D: nabpaclitaxel + durvalumab; QoL: Quality of life.

tinue to be a need for subsequent treatment options. Therefore, the promising activity [19] and QoL results of the ABOUND.2L+ trial suggest nab-paclitaxel monotherapy could be considered following disease progression.

There are some limitations to our study. First, $63-73 \%$ of patients completed baseline and $\geq 1$ postbaseline QoL assessment, which may affect the interpretation of the results. As is the case in any longitudinal QoL study, patients who continue to receive treatment and whose QoL is measured tend to be those with better outcomes, and this potential survivor selection bias needs to be considered. Second, patients were not randomized to the nabpaclitaxel + durvalumab arm, as was done for the other arms of the ABOUND.2L+ study, and certain differences 
between the arms reported here exist. Most notably, the nab-paclitaxel + durvalumab arm included patients with both squamous and nonsquamous histology, while the nab-paclitaxel alone arm only included nonsquamous histology.

\section{Conclusion}

At present, neither nab-paclitaxel monotherapy nor nab-paclitaxel + durvalumab are standards of care as subsequent therapy for NSCLC. When the ABOUND.2L+ trial was designed, pemetrexed and docetaxel were considered standards of care for subsequent therapy in NSCLC. However, an increased trend in first-line pemetrexed and potential concerns with second-line docetaxel fueled the need for more subsequent therapy options. Although nab-paclitaxel monotherapy or in combination with durvalumab are not standards of care for subsequent therapy, the primary publication of the ABOUND.2L+ trial reported encouraging outcomes with nab-paclitaxel alone [19], and the present findings suggest promising QoL trends. However, additional data are required to further support the potential use of these regimens as treatment options in this setting. Furthermore, these results may encourage future trials of additional nab-paclitaxel-based regimens in advanced NSCLC.

\section{Summary points}

- Quality of life (QoL) is an important consideration during treatment in patients with advanced non-small cell lung cancer (NSCLC).

- The aim of this analysis was to evaluate QoL in patients with advanced NSCLC who received nab-paclitaxel alone or nab-paclitaxel + durvalumab in the second or third line setting in the ABOUND.2L+ trial.

- A total of 80 and 79 patients were enrolled to receive nab-paclitaxel alone or nab-paclitaxel + durvalumab, respectively; $50(62.5 \%)$ and $58(73.4 \%)$ patients, respectively, completed a baseline and $\geq 1$ postbaseline QoL assessment.

- Lung Cancer Symptom Scale (LCSS) total score was stable through eight treatment cycles in both arms. LCSS component and composite scores were largely stable or improved. Clinically meaningful improvement was noted in the three-item index and overall constitutional score in the nab-paclitaxel + durvalumab arm. Mean maximum component scores showed clinically meaningful improvement in both the nab-paclitaxel alone (seven of nine items) and nab-paclitaxel + durvalumab (eight of nine items) arms.

- EuroQoL Five-Dimensions Five-Levels scores were stable through eight treatment cycles in both arms, except for clinically meaningful deterioration in the nab-paclitaxel alone arm beginning with cycle 8 . All domains were

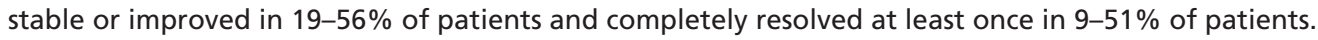

- European Organisation for Research and Treatment of Cancer Quality of Life Questionnaire 30-item core scores were stable or improved through eight treatment cycles, except for clinically meaningful deterioration in role functioning at later cycles. Mean maximum scores showed clinically meaningful improvement in emotional functioning and global health status/QoL in both arms, and in pain reduction in the nab-paclitaxel + durvalumab arm.

- QoL was generally stable or improved through eight cycles of nab-paclitaxel alone or nab-paclitaxel + durvalumab as second or third line treatment in patients with NSCLC.

\section{Financial \& competing interests disclosure}

This study was supported by Celgene Corporation, Summit, NJ, USA. SP Aix, M Cobo Dols, JR Fischer and O Juan-Vidal report nothing to disclose. D Talbot has served on an advisory board for AstraZeneca, Boehringer Ingelheim, Celgene, Pfizer, Eli Lilly, Novartis, Clovis and Roche; has been a consultant for Boehringer Ingelheim, Celgene and Achilles; and reports honoraria from Pfizer, Novartis and Celgene. R Govindan has served on an advisory board for AbbVie, AstraZeneca, Baxalta, Boehringer Ingelheim, Celgene, Merck, MSK, Pfizer and Roche; has been a consultant for AbbVie, ARIAD, Astellas, Bristol-Myers Squibb, Genentech and INC Research; and reports honoraria from AbbVie. PE Postmus has served on an advisory board for AstraZeneca, Boehringer Ingelheim, Bristol-Myers Squibb, Celgene, Clovis, Janssen, MSD and Roche. C Lewanski has been a consultant for Eli Lilly. J Bennouna has served on an advisory board for Amgen, AstraZeneca, Bristol-Myers Squibb and Novartis; and reports symposium for AstraZeneca, Novartis and Roche. DJ Stewart has served as a consultant, has served on advisory boards and has received honoraria from Roche Canada, Boehringer Ingelheim Canada, Merck Canada, AstraZeneca Canada, Bristol-Myers Squibb Canada and Exactis and clinical trials support from Boehringer Ingelheim, AstraZeneca, Novartis, Bristol-Myers Squibb and Celgene. A Ardizzoni reports honoraria from Boehringer Ingelheim, Bristol-Myers Squibb, Eli Lilly and MSD. R Bhore and TJ Ong report employment or leadership position and stock ownership at Bristol-Myers Squibb. M Wolfsteiner has served as a contracted medical reviewer for Celgene Corporation. M Reck has served on an advisory board for AstraZeneca, Boehringer Ingelheim, Bristol-Myers Squibb, Celgene, Lilly, MSD, Pfizer 
and Roche. D Morgensztern has served on an advisory board for AbbVie, Bristol-Myers Squibb, Takeda, Pharma Mar and Boehringer Ingelheim. The authors have no other relevant affiliations or financial involvement with any organization or entity with a financial interest in or financial conflict with the subject matter or materials discussed in the manuscript apart from those disclosed.

Writing assistance was provided by R Tweedell of MediTech Media, Ltd, through funding by Bristol-Myers Squibb. The authors are fully responsible for all content and editorial decisions for this manuscript.

\section{Data sharing statement}

The authors certify that this manuscript reports original clinical trial data. Data requests may be submitted to Celgene, A BristolMyers Squibb Company at https://vivli.org/ourmember/celgene/ and must include a description of the research proposal. The source of this data is: NCT02250326.

\section{Open access}

This work is licensed under the Attribution-NonCommercial-NoDerivatives 4.0 Unported License. To view a copy of this license, visit http://creativecommons.org/licenses/by-nc-nd/4.0/

\section{References}

Papers of special note have been highlighted as: $\bullet$ of interest; $\bullet \bullet$ of considerable interest

1. Polanski J, Jankowska-Polanska B, Rosinczuk J, Chabowski M, Szymanska-Chabowska A. Quality of life of patients with lung cancer. Onco. Targets Ther. 9, 1023-1028 (2016).

- Review article on quality of life (QoL) in patients with lung cancer, emphasizing the importance of patient-reported outcomes and strategies that can help improve QoL.

2. Iyer S, Roughley A, Rider A, Taylor-Stokes G. The symptom burden of non-small-cell lung cancer in the USA: a real-world cross-sectional study. Support Care Cancer 22(1), 181-187 (2014).

3. Walker MS, Wong W, Ravelo A, Miller PJE, Schwartzberg LS. Effectiveness outcomes and health related quality of life impact of disease progression in patients with advanced nonsquamous NSCLC treated in real-world community oncology settings: results from a prospective medical record registry study. Health Qual. Life Outcomes 15(1), 160 (2017).

4. Chouaid C, Agulnik J, Goker E et al. Health-related quality of life and utility in patients with advanced non-small-cell lung cancer: a prospective cross-sectional patient survey in a real-world setting. J. Thorac. Oncol. 8(8), 997-1003 (2013).

5. Abraxane ${ }^{\circledR}$ for injectable suspension (paclitaxel protein-bound particles for injectable suspension) (albumin-bound) [package insert]. Celgene Corporation, Summit, NJ, USA (2018).

6. Thomas M, Spigel DR, Jotte RM et al. Nab-paclitaxel/carboplatin induction in squamous NSCLC: longitudinal quality of life while on chemotherapy. Lung Cancer (Auckl.) 8, 207-216 (2017).

-• The Phase III ABOUND.sqm trial of first-line $n a b$-paclitaxel/carboplatin in patients with advanced squamous non-small-cell lung cancer (NSCLC), demonstrating clinically meaningful improvement in QoL with nab-paclitaxel/carboplatin; responders appeared to benefit more than nonresponders.

7. Gandhi L, Rodriguez-Abreu D, Gadgeel S et al. Pembrolizumab plus chemotherapy in metastatic non-small-cell lung cancer. N. Engl. J. Med. 378(22), 2078-2092 (2018).

8. Papadimitrakopoulou V, Cobo M, Bordoni R et al. IMpower132: PFS and safety results with $1 \mathrm{~L}$ atezolizumab+carboplatin/cisplatin+pemetrexed in stage IV non-squamous NSCLC. J. Thorac. Oncol. 13(10), S332-S333 Abstract OA05.07 (2018).

9. Socinski MA, Jotte RM, Cappuzzo F et al. Atezolizumab for first-line treatment of metastatic nonsquamous NSCLC. N. Engl. J. Med. 378(24), 2288-2301 (2018).

10. West $\mathrm{H}, \mathrm{McCleod} \mathrm{M}$, Hussein $\mathrm{M}$ et al. Atezolizumab in combination with carboplatin plus nab-paclitaxel chemotherapy compared with chemotherapy alone as first-line treatment for metastatic non-squamous non-small-cell lung cancer (IMpower130): a multicentre, randomised, open-label, Phase III trial. Lancet Oncol. 20(7), 924-937 (2019).

11. Jotte RM, Cappuzzo F, Vynnychenko I et al. IMpower131: primary PFS and safety analysis of a randomized phase III study of atezolizumab+carboplatin + paclitaxel or nab-paclitaxel vs carboplatin $+n a b$-paclitaxel as $1 \mathrm{~L}$ therapy in advanced squamous NSCLC. J. Clin. Oncol. 36(Suppl.) Abstract LBA9000 (2018).

12. Paz-Ares L, Luft A, Vicente D et al. Pembrolizumab plus chemotherapy for squamous non-small-cell lung cancer. N. Engl. J. Med. 379(21), 2040-2051 (2018).

13. Jain P, Jain C, Velcheti V. Role of immune-checkpoint inhibitors in lung cancer. Ther. Adv. Respir. Dis. 12, 1753465817750075 (2018).

14. Antonia SJ, Villegas A, Daniel D et al. Overall survival with durvalumab after chemoradiotherapy in stage III NSCLC. N. Engl. J. Med. 379(24), 2342-2350 (2018).

15. National Comprehensive Cancer Network. NCCN Guidelines ${ }^{\circledR}$. Non-small-cell lung cancer. V2.2020. (2020) https://www.nccn.org/professionals/physician_gls/pdf/nscl.pdf 
16. Yang JC, Hirsh V, Schuler M et al. Symptom control and quality of life in LUX-Lung 3: a Phase III study of afatinib or cisplatin/pemetrexed in patients with advanced lung adenocarcinoma with EGFR mutations. J. Clin. Oncol. 31(27), 3342-3350 (2013).

- The Phase III LUX-Lung 3 trial of first-line afatinib or pemetrexed/cisplatin in patients with EGFR mutation-positive NSCLC, demonstrating improved dyspnea and cough with afatinib versus chemotherapy; additionally, worsening of diarrhea, dysphagia and sore mouth with afatinib and worsening of nausea, vomiting and fatigue with chemotherapy were observed.

17. Boye $\mathrm{M}$, Wang $\mathrm{X}$, Srimuninnimit $\mathrm{V}$ et al. First-line pemetrexed plus cisplatin followed by gefitinib maintenance therapy versus gefitinib monotherapy in East Asian never-smoker patients with locally advanced or metastatic nonsquamous non-small-cell lung cancer: quality of life results from a randomized Phase III trial. Clin. Lung Cancer 17(2), 150-160 (2016).

- A Phase III trial of first-line pemetrexed/cisplatin followed by gefitinib versus gefitinib alone in East Asian never-smoker patients with advanced nonsquamous NSCLC, demonstrating better QoL outcomes with gefitinib alone; in patients with wildtype EGFR, the QoL outcomes were better with chemotherapy/gefitinib.

18. Geater SL, Xu CR, Zhou C et al. Symptom and quality of life improvement in LUX-Lung 6: an open-label Phase III study of afatinib versus cisplatin/gemcitabine in Asian patients with EGFR mutation-positive advanced non-small-cell lung cancer. J. Thorac. Oncol. 10(6), 883-889 (2015).

- The Phase III LUX-Lung 6 trial of first-line afatinib versus gemcitabine/cisplatin in Asian patients with EGFR mutation-positive advanced NSCLC, demonstrating better QoL outcomes with afatinib.

19. Morgensztern D, Cobo M, Ponce Aix S et al. ABOUND.2L+: a randomized Phase II study of nanoparticle albumin-bound paclitaxel with or without CC-486 as second-line treatment for advanced nonsquamous non-small-cell lung cancer (NSCLC). Cancer 124(24), 4667-4675 (2018).

-• The Phase II ABOUND.2L+ trial (primary study) of second-line nab-paclitaxel with/without CC-486 in patients with advanced nonsquamous NSCLC, demonstrating promising efficacy and acceptable safety profile with nab-paclitaxel; CC-486 did not offer additional benefit.

20. Govindan R, Cobo Dols M, Aix S et al. Nab-paclitaxel+durvalumab as second- or third-line treatment of advanced NSCLC: results from ABOUND.2L+. J Thorac. Oncol. 12(11), S1839-S1840 Abstract MA10.02 (2017).

21. Hollen PJ, Gralla RJ, Kris MG et al. Measurement of quality of life in patients with lung cancer in multicenter trials of new therapies. Psychometric assessment of the Lung Cancer Symptom Scale. Cancer 73(8), 2087-2098 (1994).

22. Pickard AS, De Leon MC, Kohlmann T, Cella D, Rosenbloom S. Psychometric comparison of the standard EQ-5D to a 5 level version in cancer patients. Med. Care 45(3), 259-263 (2007).

23. Niezgoda HE, Pater JL. A validation study of the domains of the core EORTC quality of life questionnaire. Qual. Life Res. 2(5), 319-325 (1993).

24. Hirsh V. Are the data on quality of life and patient reported outcomes from clinical trials of metastatic non-small-cell lung cancer important? World J. Clin. Oncol. 4(4), 82-84 (2013).

25. Reck M, Brahmer J, Bennett B et al. Evaluation of health-related quality of life and symptoms in patients with advanced non-squamous non-small-cell lung cancer treated with nivolumab or docetaxel in CheckMate 057. Eur. J. Cancer 102, 23-30 (2018).

- The Phase III CheckMate 057 trial of second-line nivolumab versus docetaxel in patients with advanced nonsquamous NSCLC, demonstrating better QoL outcomes with nivolumab versus docetaxel.

26. Vodermaier A, Lucas S, Linden W, Olson R. Anxiety after diagnosis predicts lung cancer-specific and overall survival in patients with stage III non-small-cell lung cancer: a population-based cohort study. J. Pain Symptom Manage. 53(6), 1057-1065 (2017).

27. Chambers SK, Dunn J, Occhipinti $S$ et al. A systematic review of the impact of stigma and nihilism on lung cancer outcomes. $B M C$ Cancer 12, 184 (2012).

28. Chen J, Li W, Cui L et al. Chemotherapeutic response and prognosis among lung cancer patients with and without depression. J. Cancer 6(11), 1121-1129 (2015). 